\title{
Expression profile and diagnostic value of circRNAs in peripheral blood from patients with systemic lupus erythematosus
}

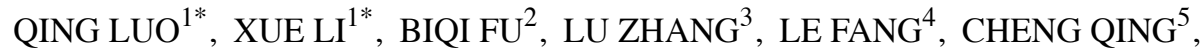 \\ YANG GUO $^{1}$, ZIKUN HUANG ${ }^{1}$ and JUNMING LI ${ }^{1}$ \\ ${ }^{1}$ Department of Clinical Laboratory, The First Affiliated Hospital of Nanchang University, Nanchang, \\ Jiangxi 330006; ${ }^{2}$ Department of Rheumatology, The First Affiliated Hospital of Nanchang University, \\ Nanchang, Jiangxi $710065 ;{ }^{3}$ Department of Medical College, Nanchang University, Nanchang, \\ Jiangxi 330006; ${ }^{4}$ Department of Blood Transfusion, 521 Hospital of Ordnance Industry, Xi'an, \\ Shanxi 710065; ${ }^{5}$ Department of Intensive Care Unit, The First Affiliated Hospital of Nanchang University, \\ Nanchang, Jiangxi 330006, P.R. China
}

Received November 5, 2019; Accepted July 28, 2020

DOI: $10.3892 / \mathrm{mmr} .2020 .11639$

\begin{abstract}
Circular RNAs (circRNAs) have gained attention due to their performance in disease diagnosis. However, the characteristics of circRNAs in peripheral blood from patients with systemic lupus erythematosus (SLE) remain unknown. Therefore, the aim of the present study was to determine the expression profile and diagnostic potential of circRNAs in peripheral blood from patients with SLE. The global circRNA expression in the peripheral blood of patients with SLE and healthy controls (HCs) was detected using a circRNA microarray. Then, the expression levels of three upregulated circRNAs were selected for further validation by reverse transcription-quantitative PCR (RT-qPCR) in a training set. Moreover, the diagnostic value of these circRNAs was assessed by constructing a receiver operating characteristic curve, and then verified in a blind testing set. In total, 1,566 circRNAs were identified to be dysregulated between patients with SLE and HCs ( $\geq 2$ fold change, $\mathrm{P}<0.05$ ). Furthermore, the RT-qPCR results were consistent with the microarray data, in that all three selected circRNAs, hsa_circ_0082688,hsa_circ_0082689 and hsa_circ_0008675, were significantly upregulated in patients with SLE $(\mathrm{P}<0.05)$. Results from the training set demonstrated that the combination of hsa_circ_0082688-hsa_circ_0082689 may provide the most beneficial diagnostic potential. Moreover, the blind test results indicated that the combination model
\end{abstract}

Correspondence to: Dr Junming Li, Department of Clinical Laboratory, The First Affiliated Hospital of Nanchang University, 17 Yongwai Zheng Jie, Nanchang, Jiangxi 330006, P.R. China E-mail: lisir361@163.com

${ }^{*}$ Contributed equally

Key words: systemic lupus erythematosus, circular RNAs, microarray assay, peripheral blood of hsa_circ_0082688-hsa_circ_0082689 could effectively discriminate between patients with SLE from patients with rheumatoid arthritis and $\mathrm{HCs}$, with a sensitivity of $91.30 \%$, a specificity of $78.57 \%$ and an accuracy of $82.28 \%$. Moreover, the combination model of hsa_circ_0082688-hsa_circ_0082689 + anti-dsDNA could more effectively discriminated the SLE group from the control groups, with a sensitivity of $95.65 \%$, a specificity of $100.00 \%$ and an accuracy of $98.73 \%$. In addition, correlation analysis results suggested that all three circRNAs in patients with SLE did not correlate with the SLE disease activity index. In conclusion, the expression levels of hsa_circ_0082688-hsa_circ_0082689 may serve as potential biomarkers for SLE diagnosis.

\section{Introduction}

Systemic lupus erythematosus (SLE) is an autoimmune disease characterized by the production of auto-antibodies, forming immune complexes and potentially causing life-threatening renal, cardiac or brain damage $(1,2)$. Due to the heterogeneous clinical manifestations and unpredictable disease course, accurate diagnosis is important for correct treatment and good prognosis of patients with SLE; however, the accurate diagnosis of SLE is difficult due to this heterogeneity of clinical manifestations and the ambiguity of the pathogenesis (3-6). Currently, there is a lack of sensitive and specific diagnostic methods for SLE, but there have been an increased number of studies investigating novel biomarkers for improved SLE diagnosis (7-9).

Circular RNAs (circRNAs) are a type of closed circular non-coding RNA $(10,11)$. Moreover, as circRNAs do not have 5' or 3 ' ends, they are resistant to exonuclease-mediated degradation and are more stable compared with most linear RNAs (12). Recently, circRNAs have received increasing interest due to their potential in regulating gene expression, mainly by acting as 'microRNA (miRNA/miR) sponges' to sequester target miRNAs (13-15). Furthermore, aberrant expression of circRNAs has been revealed to occur in numerous diseases, 
such as atherosclerotic vascular disease, neurological disorders, prion diseases, cancer and autoimmune diseases (16-19). While the 'sponge' function of circRNAs has been the focus of research, several other circRNA roles have also been studied.

Previous studies have reported that high levels of circRNAs are widely distributed in the cytoplasm, nucleus and a variety of body fluids, including saliva and blood $(20,21)$, and often demonstrate tissue and developmental stage-specific expression $(13,22,23)$. Due to their high abundance, stability, tissue-specific expression and easily availability, circRNAs possess the potential to serve as biomarkers for diseases diagnosis. For example, Zhao et al (24) revealed that hsa_circ_0054633 in peripheral blood could be used as a new biomarker for pre-diabetes and type 2 diabetes mellitus. Moreover, Zhao et al (25) identified that peripheral blood hsa_circ_0124644 can be used as a diagnostic biomarker of coronary artery disease.

Our previous study showed that there are several differentially expressed circRNAs in peripheral blood mononuclear cells (PBMCs) between patients with SLE and healthy controls $(\mathrm{HCs})$, and that certain differentially expressed circRNAs may have roles in the pathogenesis of SLE (26). Therefore, the aim of the present study was to assess the potential of circRNAs as biomarkers for SLE diagnosis in peripheral blood samples, which is a sample that is relatively simple for collection and preprocessing.

\section{Materials and methods}

Patients and ethics statement. Patients who fulfilled the revised American College of Rheumatology criteria for SLE (27) were recruited from The First Affiliated Hospital of Nanchang University between November 2016 and September 2017. Disease activity was assessed using the SLE disease activity index (SLEDAI) (28). Patients with SLE were classified into an inactive group (SLEDAI, 0-9) and an active group (SLEDAI, $\geq 10$ ) according to the SLEDAI (28). Healthy volunteers unrelated to the patients with SLE and who had no inflammatory or autoimmune diseases were recruited as HCs. As an autoimmune disease control, patients with rheumatoid arthritis (RA) who fulfilled the revised American College of Rheumatology criteria for RA (29) were enrolled from The First Affiliated Hospital of Nanchang University between November 2016 and September 2017. The samples for this study were stored (immediately after collection) in the Department of Clinical Laboratory, The First Affiliated Hospital of Nanchang University.

This study was approved by the Ethics Committee of The First Affiliated Hospital of Nanchang University (approval no. 2014003) and was performed in compliance with the Helsinki Declaration (30).

Samples collection and total RNA extraction. Blood sample collection was performed as follows: After overnight fasting, $2 \mathrm{ml}$ blood was collected from the median cubital vein of each subject and then stored in EDTA anticoagulant vacutainers. Total RNA was extracted within $4 \mathrm{~h}$ using TRIzol ${ }^{\circledR}$ (Invitrogen, Inc.; Thermo Fisher Scientific, Inc.) reagent according to the manufacturer's instruction. The concentration and quality of the RNA were assessed by absorbance spectrometry measuring absorbance ratios of A260/A280 and A260/A230, respectively, using a NanoDrop ND-1,000 spectrophotomete (Agilent Technologies, Inc.).

Microarray analysis. Equal amounts of RNA from three patients with SLE were collected in a SLE sample for microarray experiment. Equal amounts of RNA from three HCs were also collected in a HC sample for microarray experiment. Sample labeling and array hybridization were performed according to the manufacturer's protocol (Arraystar, Inc.). Total RNA was digested with RNase R (Epicentre, Inc.) to remove linear RNAs and enrich circRNAs. The enriched circRNAs were amplified and transcribed into fluorescent circRNAs using a random priming method (Arraystar Super RNA Labeling kit; Arraystar, Inc.). Then, the labeled circRNAs were hybridized to the Arraystar Human circRNA Microarray (version 2.0; $8 \times 15 \mathrm{~K}$; Arraystar, Inc). After washing the slides, the arrays were scanned with an Agilent Scanner G2505C (Agilent Technologies, Inc.). Then, Agilent Feature Extraction software (version 11.0.1.1) (Agilent Technologies, Inc.) (19) was used to analyze the acquired array images. Quantile normalization and subsequent data processing were performed using $\mathrm{R}$ software package ( $\mathrm{R}$ version 3.1.2) (Agilent Technologies, Inc.) (19). Ggplot2 (version R-3.3.2; r-project.org/) was used to create a heat map. The microarray work was performed by KangChen Bio-Tech (Shanghai, China).

Reverse transcription-quantitative PCR (RT-qPCR) analysis. Total RNA was reverse transcribed into cDNA using a PrimeScript $^{\mathrm{TM}}$ RT reagent kit (Takara Bio, Inc.). The RT reaction was performed in a $10 \mu 1$ reaction containing $5 \mathrm{X}$ PrimeScriptTM Buffer, $1.0 \mu \mathrm{l}$ RT specific primer, $0.5 \mu 1$ PrimeScriptTM RT Enzyme Mix and $5.0 \mu \mathrm{g}$ total RNA. The RT assay was set at an initial denaturation step at $37^{\circ} \mathrm{C}$ for $15 \mathrm{~min}$, followed by $85^{\circ} \mathrm{C}$ for $5 \mathrm{sec}$. qPCR was then performed on an ABI 7,500 RT PCR system (Applied Biosystems; Thermo Fisher Scientific, Inc.), using SYBR ${ }^{\circledR}$ Premix Ex $\mathrm{Taq}^{\mathrm{TM}}$ II (Takara Bio, Inc.). The following PCR conditions were used: Initial denaturation at $95^{\circ} \mathrm{C}$ for $5 \mathrm{~min}$, followed by 40 cycles at $95^{\circ} \mathrm{C}$ for $15 \mathrm{sec}$ and $60^{\circ} \mathrm{C}$ for $1 \mathrm{~min}$, then a melt curve was detected to assess the specificity of amplification and lack of primer dimers. The primers used for RT-qPCR are presented in Table I. $\beta$-actin was used as an internal control. After the reactions, the $\mathrm{Cq}$ values were determined using the fixed threshold settings. The relative expression of circRNAs was calculated using the $2^{-\Delta \Delta \mathrm{Cq}}$ method (31) normalized to endogenous control, with $\Delta \mathrm{Cq}=\mathrm{Cq}_{\text {target }}-\mathrm{Cq}_{\text {reference. }}$.

Blood routine, serum inflammatory indicators and autoantibodies determination. The concentrations of serum $\mathrm{C} 3, \mathrm{C} 4$ and $\mathrm{C}$-reactive protein (CRP) were determined by nephelometry methods, according to the manufacturer's instructions (IMMUNE800; Beckman Coulter, Inc.). Erythrocyte sedimentation rate (ESR) and blood routine were also determined according to the manufacturer's instructions.

The antinuclear antibody was detected using an indirect immunofluorescence method according to the manufacturer's instructions (Euroimmun AG). Anti-dsDNA of $\operatorname{IgG}$ in serum was determined by using both an indirect immunofluorescence method (Euroimmun AG) and ELISA kits (cat. 
Table I. Specific circRNA primers used for reverse transcription-quantitative PCR.

\begin{tabular}{ll}
\hline Name & \multicolumn{1}{c}{ Sequence } \\
\hline hsa_circ_0008675 & Forward: 5'-GGAAGCCTTGCAGTTTGCTC-3' \\
hsa_circ_0082689 & Reverse: 5'-AGCATTGGCTGGTGGGTTAT-3' \\
& Forward: 5'-GTCCCCAAACACTCTTAGCCA-3' \\
hsa_circ_0082688 & Reverse: 5'-CACACTCAGGTTGTGTTCGG-3' \\
$\beta$-actin & Forward: 5'-TGCCGTATCGATGGCAATTC-3' \\
& Reverse: 5'-ATAGCTCAGGTGGTCAACGC-3' \\
& Forward: CATGTACGTTGCTATCCAGGC \\
& Reverse: CTCCTTAATGTCACGCACGAT
\end{tabular}

circRNA, circular RNA.

no. KX-E-DSD01096; Shanghai Kexin Biotech Co., Ltd.). Anti-extractable nuclear antigens (ENAs) antibodies including anti-sjögren syndrome A antigen antibody (anti-SSA), anti-sjögren syndrome B antigen antibody (anti-SSB), anti-Ro52, anti-Smith antibody (anti-Sm), anti-nuclear ribonucleoprotein/Smith antibody (anti-nRNP/Sm), anti-ribosomal protein $\mathrm{P}$ (anti-RIB-P), anti-histone and anti-nucleosome antibody were determined using line immunoassays kits (cat. no. DL 1590-6401-3G; Euroimmun AG) according to the manufacturer's instructions. The results of anti-ENAs detection were presented as negative $(-)$ or positive $(+,++,+++)$ using EuroBlot One (Euroimmun AG).

Statistical analysis. Statistical analysis and graphic presentation were conducted with GraphPad Prism version 5.0 (GraphPad Software, Inc.) and SPSS version 16.0 (IBM Corp.). Student's t-test was used with normalized data, while the non-parametric Mann-Whitney test was used to analyze data that did not pass the normality test. A Kruskal-Wallis test was used in comparisons between three groups and Dunns post hoc test was used following the Kruskal-Wallis test. Moreover, the Pearson's method or the non-parametric Spearman's method were used for correlation analysis. The cut-off values were determined by receiver operating characteristic (ROC) curves analysis using GraphPad Prism version 5.0; ROC curves were performed to evaluate the diagnostic value of circRNAs that were dysregulated in the peripheral blood of patients with SLE compared to HCs. A parallel model was used to evaluate the diagnostic efficiency of the hsa_circ_0082688-hsa_circ_0082689 combination model (32); if one of the multiple indicators is positive, the disease can be diagnosed. $\mathrm{P}<0.05$ was considered to indicate a statistically significant difference.

\section{Results}

Characteristics of the study population. A total of 185 participants were enrolled in the present study, including 76 patients with SLE, $76 \mathrm{HCs}$ and 33 patients with RA. Among the patients with SLE, 13 were newly diagnosed patients with no history of corticosteroids or immunosuppressive drugs use before registration. The demographic characteristics of the study population are presented in Tables II and III.

In the discovery set, there were three patients with newly diagnosed SLE and three age-matched and sex-matched HCs. An additional 50 patients with SLE and $50 \mathrm{HCs}$ were included in the training set for the validation of differentially expressed circRNAs and diagnostic model construction. In this patient set, ten patients had newly diagnosed SLE with no history of corticosteroids or immunosuppressive drugs use before registration. Moreover, the other patients with SLE were recurrent patients and had received therapy with corticosteroids or immunosuppressive drugs for $\geq 1$ month before registration. An independent cohort consisting of 23 patients with SLE, 33 patients with RA and 23 HCs were enrolled in the blind testing set for clinical evaluation of circRNAs in SLE diagnosis. It was demonstrated that there were no significant differences in age and sex between the SLE and HCs groups. Moreover, there were no significant differences in sex between the RA, SLE and HCs groups. Due to the difference in the ages of high incidence occurrence between RA and SLE (the incidence of RA is high in individuals who are 50-60 years, while the incidence of SLE is high in 20-40 years), patients with RA and SLE were not age-matched in the present study.

CircRNA expression profiling in peripheral blood from patients with SLE. The overall distribution of microarray data of these two groups is presented as a box plot (Fig. 1A) and scatter plot (Fig. 1B), and significant differences in the expression levels of circRNAs between patients with SLE and HCs were screened with $>2.0$ fold change and $\mathrm{P}<0.05$. The results indicated that, compared with the HC group, 753 circRNAs were significantly upregulated, while 813 circRNAs were significantly downregulated in the SLE group. Furthermore, a heat map was created to group the circRNAs based on their expression levels among the samples (Fig. 1C).

Validation of circRNA expression in the training set. As the main objective of the study was to identify diagnostic markers of SLE in peripheral blood, the focus was on the upregulated circRNAs in patients with SLE. To assess the microarray data, three circRNAs (hsa_circ_0082688, hsa_circ_0082689 and hsa_circ_0008675) that were not only listed in the top 50 most 

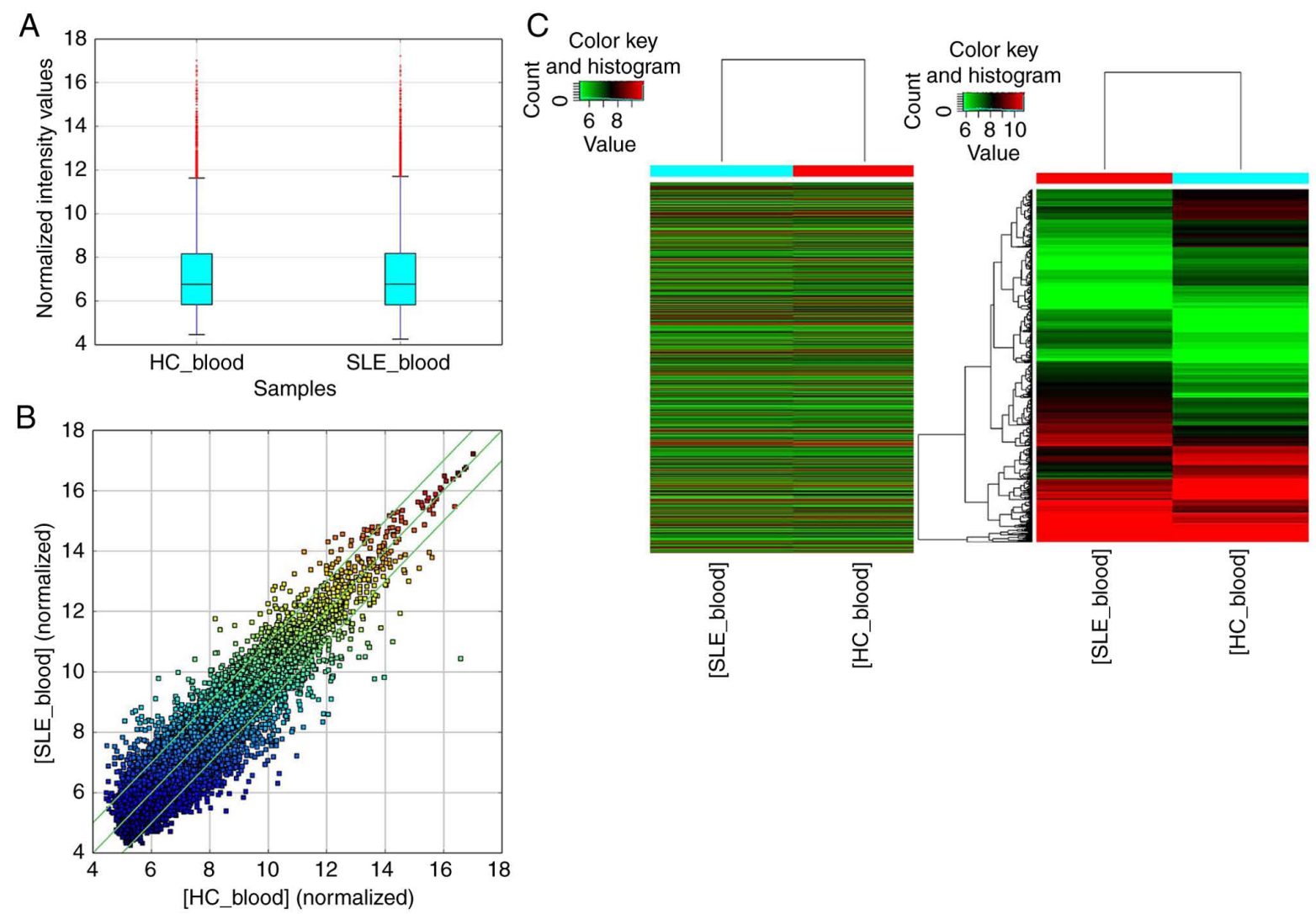

Figure 1. Microarray assay results demonstrate the circRNA expression profiles in peripheral blood from three patients with SLE and 3 HCs. (A) Box plot used to visualize the distributions of a dataset for the circRNAs profiles. (B) circRNA scatter plot. Dots above the top green line and below the bottom green line indicate $>1.5$-fold of changes of logarithmized circRNAs between the two groups. (C) Heat map of differentially expressed circRNAs. circRNAs, circular RNAs; HC, healthy controls; SLE, systemic lupus erythematosus.

Table II. Demographic characteristics of the study population.

\begin{tabular}{|c|c|c|c|c|}
\hline Study set & Categories & SLE & $\mathrm{HC}$ & RA \\
\hline \multirow[t]{4}{*}{ Discovery set } & & 3 & 3 & \\
\hline & Females & $3(100.00)$ & $3(100.00)$ & \\
\hline & Age, years & $29.00 \pm 15.40$ & $34.00 \pm 7.21$ & \\
\hline & SLEDAI score & $18.33 \pm 3.79^{\mathrm{a}}$ & - & \\
\hline \multirow[t]{4}{*}{ Training set } & & 50 & 50 & \\
\hline & Females & $46(92.00)$ & $43(86.00)$ & \\
\hline & Age, years & $42.70 \pm 14.80$ & $44.77 \pm 12.90$ & \\
\hline & SLEDAI score & $7.69 \pm 4.60$ & - & \\
\hline \multirow[t]{4}{*}{ Blind testing set } & & 23 & 23 & 33 \\
\hline & Females & $21(91.30)$ & $18(78.26)$ & 24 (72.73) \\
\hline & Age, years & $35.70 \pm 12.52$ & $41.30 \pm 12.70$ & $58.00 \pm 10.15^{\mathrm{b}}$ \\
\hline & SLEDAI score & $5.90 \pm 3.94$ & - & - \\
\hline
\end{tabular}

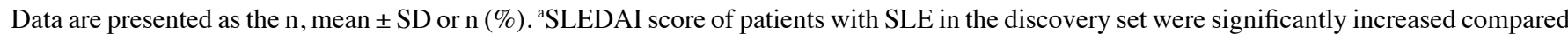
with the training set and blind testing set $($ all $\mathrm{P}<0.001)$. ${ }^{\mathrm{b}} \mathrm{Age}$ of patients with RA were significantly increased compared with SLE and HC groups (all $\mathrm{P}<0.05$ ). HC, healthy controls; RA, rheumatoid arthritis; SLE, systemic lupus erythematosus; SLEDAI, SLE disease activity index.

significant upregulated circRNAs in patients with SLE in this circRNA microarray, but also identified to be upregulated in PBMCs from patients with SLE in our previous study (26), were selected for validation by RT-qPCR in the training set, which included 50 patients with SLE and $50 \mathrm{HC}$. The results of RT-qPCR were consistent with the circRNA microarray data, in that all three circRNAs were significantly upregulated in the SLE group (Fig. 2). Moreover, the expression levels of circRNAs between patients with newly diagnosed SLE or recurrent SLE patients were also compared, but no significant 
Table III. Clinical characteristics of patients with SLE.

\begin{tabular}{|c|c|}
\hline Categories & Patients with SLE (n \\
\hline Females & $70(90.91)$ \\
\hline Age, years & $40.00 \pm 14.61$ \\
\hline SLEDAI score (67 patients) & $7.61 \pm 4.98$ \\
\hline ds-DNA, IU/ml (71 patients) & $243.80 \pm 407.82$ \\
\hline \multicolumn{2}{|l|}{ Anti-ENA (68 patients) } \\
\hline Anti-Sm & $21(30.88)$ \\
\hline Anti-Ro52 & $45(66.18)$ \\
\hline Anti-nRNP/Sm & $33(48.53)$ \\
\hline Anti-RIB-P & $26(38.24)$ \\
\hline Anti-nucleosome & $22(32.35)$ \\
\hline Anti-SSA & $44(64.71)$ \\
\hline Anti-SSB & $11(16.18)$ \\
\hline Anti-histone & $22(32.35)$ \\
\hline C3, g/1 (71 patients) & $0.62 \pm 0.20$ \\
\hline C4, g/l (71 patients) & $0.14 \pm 0.06$ \\
\hline IgG, g/l (68 patients) & $13.65 \pm 5.29$ \\
\hline ESR, mm/h (65 patients) & $35.49 \pm 34.80$ \\
\hline CRP, mg/l (71 patients) & $12.31 \pm 23.30$ \\
\hline $\mathrm{WBC}, 10^{9} / 1$ & $6.78 \pm 3.61$ \\
\hline $\mathrm{RBC}, 10^{12} / 1$ & $3.96 \pm 0.82$ \\
\hline $\mathrm{HGB}, \mathrm{g} / 1$ & $113.12 \pm 25.61$ \\
\hline $\mathrm{HCT}, 1 / 1)$ & $0.34 \pm 0.07$ \\
\hline PLT, $10^{9} / 1$ & $214.77 \pm 85.97$ \\
\hline Lymphocytes, $10^{9} / 1$ & $1.56 \pm 1.10$ \\
\hline Monocytes, $10^{9} / 1$ & $0.47 \pm 0.27$ \\
\hline Neutrophils, $10^{9} / 1$ & $4.60 \pm 2.94$ \\
\hline \multicolumn{2}{|l|}{ Clinical features } \\
\hline Fever (74 patients) & $7(9.50)$ \\
\hline $\begin{array}{l}\text { Cutaneous manifestations } \\
\text { ( } 74 \text { patients) }\end{array}$ & $12(16.22)$ \\
\hline Arthritis (74 patients) & $13(17.57)$ \\
\hline Effusion (74 patients) & $11(14.86)$ \\
\hline Hematuresis (68 patients) & $14(20.59)$ \\
\hline Pyuria (68 patients) & $10(14.71)$ \\
\hline Proteinuria (68 patients) & $25(36.76)$ \\
\hline
\end{tabular}

Data are presented as the mean \pm SD or $\mathrm{n}(\%)$. Anti-dsDNA, anti double-stranded DNA; Anti-ENA, anti extractable nucleat antigen; C3, complement 3; C4, complement 4; CRP, C-reactive protein; ESR, erythrocyte sedimentation rate; HCT, hematocrit; HGB, hemoglobin; IgG, immunoglobulin G; PLT, platelets; RBC, red blood cell; RiB-P, ribosomal protein $\mathrm{P}$; rRNP, ribosomal RNP; SLE, systemic lupus erythematosus; SLEDAI, SLE disease activity index; WBC, white blood cell.

difference was demonstrated (Fig. 2). In addition, there was no correlation between circRNAs expression levels and age or sex in the SLE, RA and HC groups (data not shown).

ROC curve analysis of identified peripheral blood circRNAs among patients with SLE. To further evaluate the potential value of the three circRNAs (hsa_circ_0082688, hsa_circ_0082689 and hsa_circ_0008675) in SLE diagnosis, ROC curve analysis was performed. The area under the curve (AUC) values demonstrated that the levels of hsa_circ_0082688,hsa_circ_0082689 and hsa_circ_0008675 in peripheral blood could separate the patients with SLE from the HCs. Moreover, the highest AUC was identified for hsa_circ_0082689 (AUC: 0.733, 95\% CI, 0.634-0.832, P<0.0001, Sensitivity: $54.0 \%$, Specificity: $88.0 \%$, Cut-off: 0.0029), followed by hsa_circ_0082688 (AUC: 0.714, 95\% CI, 0.613-0.815, P<0.0001, Sensitivity: 78.0\%, Specificity: $60.0 \%$, Cut-off: 0.0032) and hsa_circ_0008675 (AUC: 0.643, 95\% CI, 0.534-0.752, $\mathrm{P}=0.0140$, Sensitivity: $54.0 \%$, Specificity: 76.0\%, Cut-off: 0.0096; Fig. 3A).

To evaluate the cumulative performances of the three circRNAs in discriminating SLE from HC, a binary logistic regression was performed. The logistic regression model indicated that the combination of hsa_circ_0082688 and hsa_circ_0082689 could provide the best diagnostic accuracy, with an AUC of 0.740 (95\% CI, 0.642-0.838, $\mathrm{P}<0.0001$; Fig. 3B). Furthermore, the combination of all these three circRNAs (Fig. 3B) and any two circRNAs (data no shown) had no improvement in SLE diagnosis, compared with the aforementioned combination of the two circRNAs.

The diagnostic efficiency of the hsa_circ_0082688-hsa_ circ_0082689 combination model was then evaluated using the paralleling model, according to their optimal cutoff value (hsa_circ_0082688: 0.0032, hsa_circ_0082689: 0.0029). As shown in Table IV, it was demonstrated that the combination model of hsa_circ_0082688-hsa_circ_0082689 could effectively discriminate patients with SLE from HCs, with a sensitivity of $86.00 \%(43 / 50)$, a specificity of $88.00 \%(44 / 50)$ and an accuracy of $87.0 \%(87 / 100)$.

Blind test of the diagnostic value of differentially expressed circRNAs. To further evaluate the value of hsa circ_0082688-hsa_circ_0082689 combination model in SLE diagnosis, an independent cohort consisting of 23 patients with SLE, 33 patients with RA and 23 HCs were enrolled and their circRNAs expression levels were measured. Similar to the training set, it was identified that the expression levels of hsa_circ_0082688 and hsa_circ_0082689 were increased significantly in patients with SLE compared with RA and HC groups (all $\mathrm{P}<0.05$; Fig. 4). According to the optimal cutoff value found in the training stage, As shown in Table V, the combination model of hsa_circ_0082688-hsa_circ_0082689 could effectively discriminate between the SLE group and the other two control groups, with a sensitivity of $91.30 \%$ $(21 / 23)$, a specificity of $78.57 \%(44 / 56)$ and an accuracy of $82.28 \%$ (65/79). Furthermore, this diagnostic model presented a sensitivity of $91.30 \%(21 / 23)$, a specificity of $78.79 \%(26 / 33)$ and an accuracy of $83.93 \%(47 / 56)$ in discriminating the SLE group from the RA group, and a sensitivity of $91.30 \%(21 / 23)$, a specificity of $78.26 \%(18 / 23)$ and an accuracy of $84.78 \%$ (39/46) in discriminating the SLE group from the HC group.

Anti-dsDNA is a traditional and most commonly used diagnostic marker for SLE (33). The aforementioned results demonstrated that the hsa_circ_0082688-hsa_circ_0082689 combination model may be used as a novel biomarker for the diagnosis of SLE. Thus, the present study evaluated the value of hsa_circ_0082688-hsa_circ_0082689 + anti-dsDNA combination model in SLE diagnosis. According to the optimal 
Table IV. Diagnostic efficiency of the hsa_circ_0082688-hsa_circ_0082689 combination model by the parallel model in the training set.

\begin{tabular}{|c|c|c|c|c|c|}
\hline Category & $\begin{array}{c}\text { hsa_circ_0082688 }>0.0032 \text { or } \\
\text { hsa_circ_0082689 }>0.0029\end{array}$ & $\begin{array}{c}\text { hsa_circ_0082688 }<0.0032 \text { or } \\
\text { hsa_circ_0082689 }<0.0029\end{array}$ & Sensitivity & Specificity & Accuracy \\
\hline SLE (50) & 43 & 7 & $86.00 \%(43 / 50)$ & $88.00 \%(44 / 50)$ & $87.0 \%(87 / 100)$ \\
\hline $\mathrm{HC}(50)$ & 6 & 44 & & & \\
\hline
\end{tabular}

HC, healthy control; SLE, systemic lupus erythematosus.

Table V. Diagnostic efficiency of the hsa_circ_0082688-hsa_circ_0082689 combination model by the parallel model in blind testing set.

\begin{tabular}{|c|c|c|c|c|c|}
\hline Category & $\begin{array}{c}\text { hsa_circ_0082688 } \\
>0.0032 \text { or hsa_circ_- } \\
0082689>0.0029\end{array}$ & $\begin{array}{c}\text { hsa_circ_0082688 } \\
<0.0032 \text { or hsa_circ_- } \\
0082689<0.0029\end{array}$ & Sensitivity & Specificity & Accuracy \\
\hline \multicolumn{6}{|c|}{ SLE vs. RA + HC } \\
\hline SLE (23) & 21 & 2 & $91.30 \%(21 / 23)$ & $78.57 \%(44 / 56)$ & $82.28 \%(65 / 79)$ \\
\hline $\mathrm{RA}+\mathrm{HC}(56)$ & 12 & 44 & & & \\
\hline \multicolumn{6}{|l|}{ SLE vs. RA } \\
\hline SLE (23) & 21 & 2 & $91.30 \%(21 / 23)$ & $78.79 \%(26 / 33)$ & $83.93 \%(47 / 56)$ \\
\hline RA (33) & 7 & 26 & & & \\
\hline \multicolumn{6}{|l|}{ SLE vs. HC } \\
\hline SLE (23) & 21 & 2 & $91.30 \%(21 / 23)$ & $78.26 \%(18 / 23)$ & $84.78 \%(39 / 46)$ \\
\hline $\mathrm{HC}(23)$ & 5 & 18 & & & \\
\hline
\end{tabular}

HC, healthy control; RA, rheumatoid arthritis; SLE, systemic lupus erythematosus.

Table VI. Diagnostic efficiency of the hsa_circ_0082688-hsa_circ_0082689 + anti-dsDNA combination model by the parallel model in blind testing set.

\begin{tabular}{|c|c|c|c|c|c|}
\hline Category & Positive & Negative & Sensitivity & Specificity & Accuracy \\
\hline \multicolumn{6}{|c|}{ hsa_circ_0082688 $>0.0032$ or hsa_circ_0082689 $>0.0029$ or anti-dsDNA $>100$} \\
\hline SLE (23) & 22 & 1 & $95.65 \%(22 / 23)$ & $100.00 \%(56 / 56)$ & $98.73 \%(78 / 79)$ \\
\hline $\mathrm{RA}+\mathrm{HC}(56)$ & 0 & 56 & & & \\
\hline \multicolumn{6}{|c|}{ anti-dsDNA>100 } \\
\hline SLE (23) & 11 & 12 & $47.83 \%(11 / 23)$ & $100.00 \%(56 / 56)$ & $79.85 \%(67 / 79)$ \\
\hline $\mathrm{RA}+\mathrm{HC}(56)$ & 0 & 56 & & & \\
\hline
\end{tabular}

HC, healthy control; RA, rheumatoid arthritis; SLE, systemic lupus erythematosus.

cutoff value of hsa_circ_0082688-hsa_circ_0082689 identified in the aforementioned results (hsa_circ_0082688>0.0032, hsa_circ_0082689>0.0029), As shown in Table VI, the hsa circ_0082688-hsa_circ_0082689 + anti-dsDNA combination model could effectively discriminated the SLE group from the control groups (RA + HC), with a sensitivity of $95.65 \%(22 / 23)$, a specificity of $100.00 \%(56 / 56)$ and an accuracy of $98.73 \%$ (78/79) in distinguishing the patients with SLE from both control groups. Moreover, the sensitivity, specificity and accuracy of hsa_circ_0082688-hsa_circ_0082689 + anti-dsDNA combination model were increased compared with hsa_circ_0082688-hsa_circ_0082689 [sensitivity $=91.30 \%$ $(21 / 23)$, specificity $=78.57 \%(44 / 56)$, accuracy $=82.28 \%(65 / 79)]$ (Table V) and anti-dsDNA [sensitivity=47.83\% (11/23), specificity=100.00\% (56/56), accuracy=79.85\% (67/79)] (Table VI).

Association of hsa_circ_0082688,hsa_circ_0082689 and hsa_circ_0008675 expression levels in peripheral blood with SLE clinical characteristics. To determine whether 

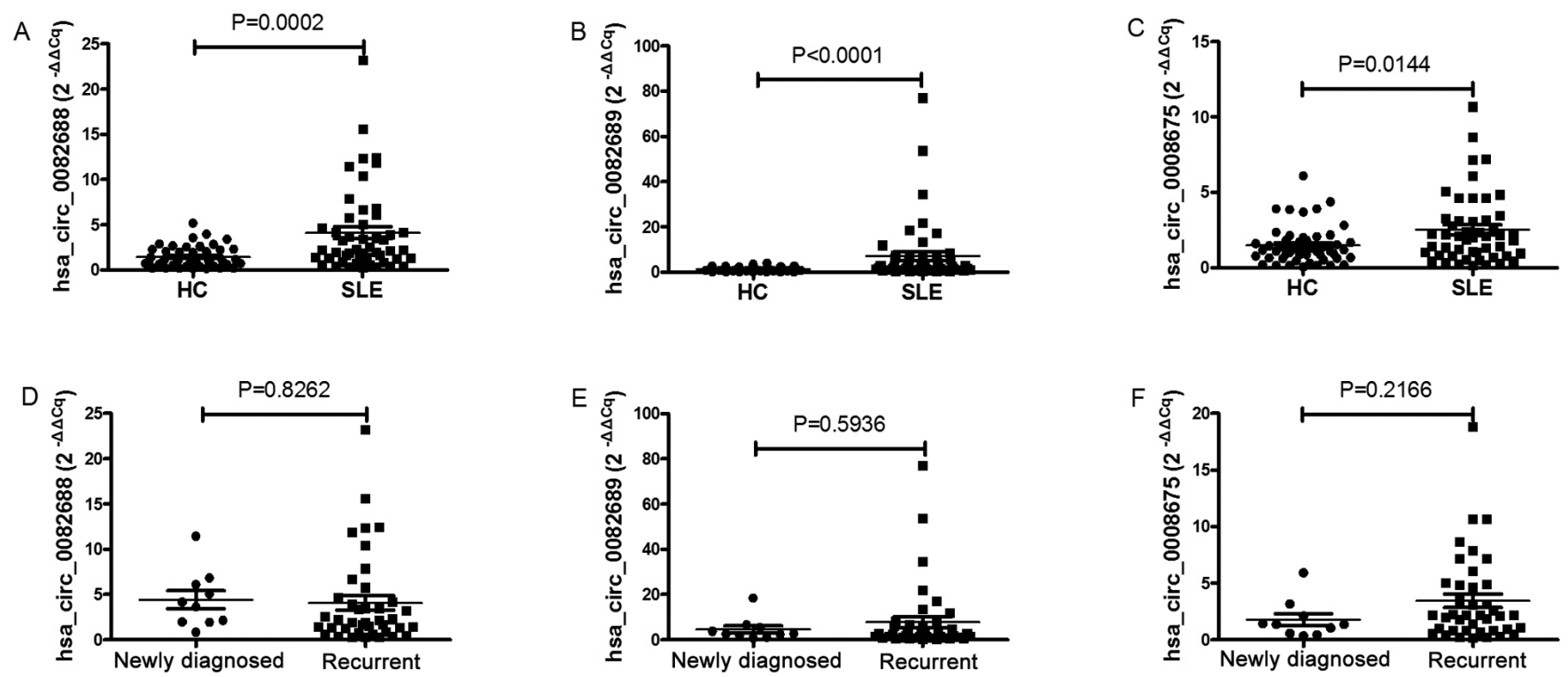

Figure 2. Reverse transcription-quantitative PCR results of the relative expression levels of circRNAs in peripheral blood from patients with SLE and the comparison group. (A) Average expression levels of hsa_circ_0082688 in patients with SLE were significantly increased compared with the HCs. (B) Average expression levels of hsa_circ_0082689 in patients with SLE were significantly increased compared with the HCs. (C) The average expression levels of hsa_circ_0008675 in patients with SLE were significantly increased compared with the HCs. (D) Average expression of hsa_circ_0082688 did not show any significant differences between patients with newly diagnosed or recurrent SLE. (E) Average expression of hsa_circ_0082689 did not show any significant differences between patients with newly diagnosed or recurrent SLE. (F) Average expression of hsa_circ_0008675 did not show any significant differences between patients with newly diagnosed or recurrent SLE. circRNAs, circular RNAs; HC, healthy controls; SLE, systemic lupus erythematosus.
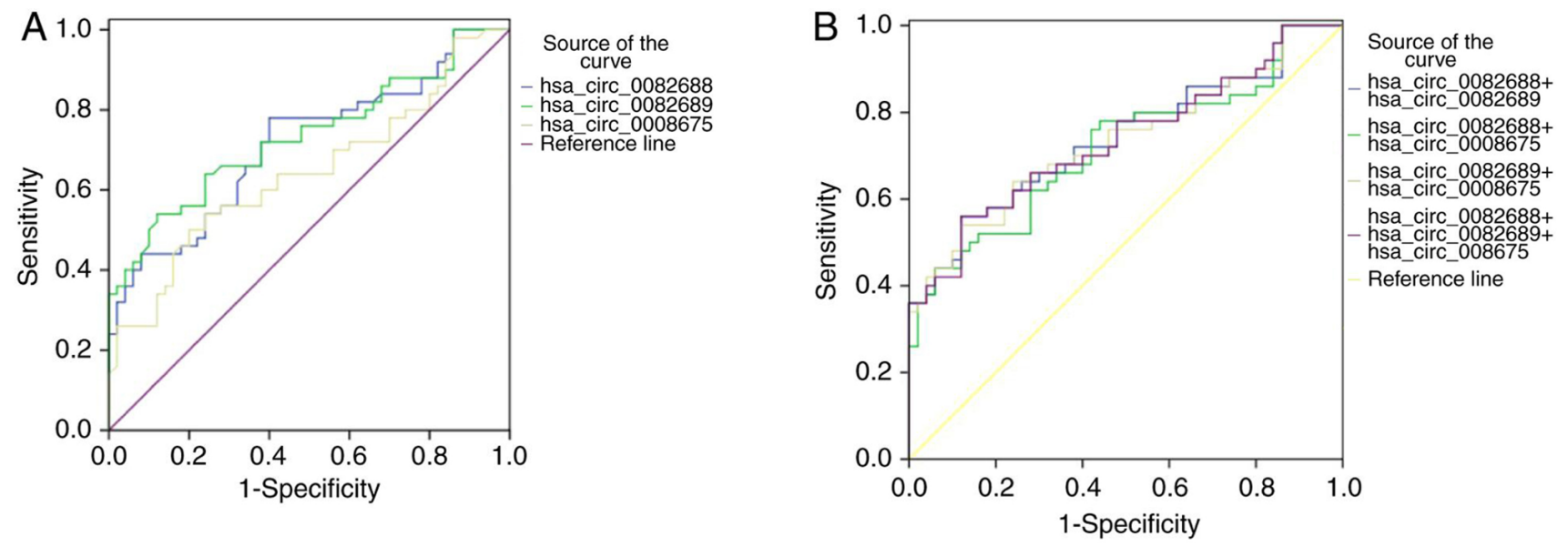

\begin{tabular}{|c|c|c|c|c|c|c|c|c|c|c|c|c|}
\hline Variables & AUC & SEM & P-value & $95 \% \mathrm{Cl}$ & Sensitivity & Specificity & Cut-off & Variables & AUC & SEM & P-value & $95 \% \mathrm{Cl}$ \\
\hline hsa_circ_0082688 & 0.714 & 0.051 & $<0.0001$ & $0.613-0.815$ & 0.78 & 0.60 & 0.0032 & hsa_circ_0082688+hsa_circ_0082689 & 0.740 & 0.050 & $<0.0001$ & $0.642-0.838$ \\
\hline hsa_dirc_0082689 & 0.733 & 0.051 & $<0.0001$ & $0.634-0.832$ & 0.54 & 0.88 & 0.0029 & hsa_circ_0082688+hsa_circ_0008675 & 0.718 & 0.052 & $<0.0001$ & $0.616-0.819$ \\
\hline \multirow[t]{2}{*}{ hsa_circ_0008675 } & 0.643 & 0.056 & 0.014 & $0.534-0.752$ & 0.54 & 0.76 & 0.0096 & hsa_circ_0082689+hsa_circ_0008675 & 0.733 & 0.051 & $<0.0001$ & $0.634-0.832$ \\
\hline & & & & & & & & $\begin{array}{l}\text { hsa_circ_0082688+hsa_circ_0082689 } \\
\text { thsa_circ_0008675 }\end{array}$ & 0.736 & 0.050 & $<0.0001$ & $0.637-0.834$ \\
\hline
\end{tabular}

Figure 3. ROC analysis of identified circRNAs in peripheral blood from patients with SLE. (A) ROC curves for circRNAs, largest AUC was demonstrated for hsa_circ_0082689, followed by hsa_circ_0082688, hsa_circ_0008675. (B) ROC curves for circRNAs in combination, combined AUC from these circRNAs (hsa_circ_0082688 and hsa_circ_0082689) was 0.740. AUC, area under the curve; circRNAs, circular RNAs; ROC, receiver operating characteristic; SLE, systemic lupus erythematosus.

the aforementioned differentially expressed circRNAs in the peripheral blood could serve as relevant biomarkers for the severity of SLE, the clinical indicators related to inflammation were collected and the SLEDAIs of all patients with SLE 

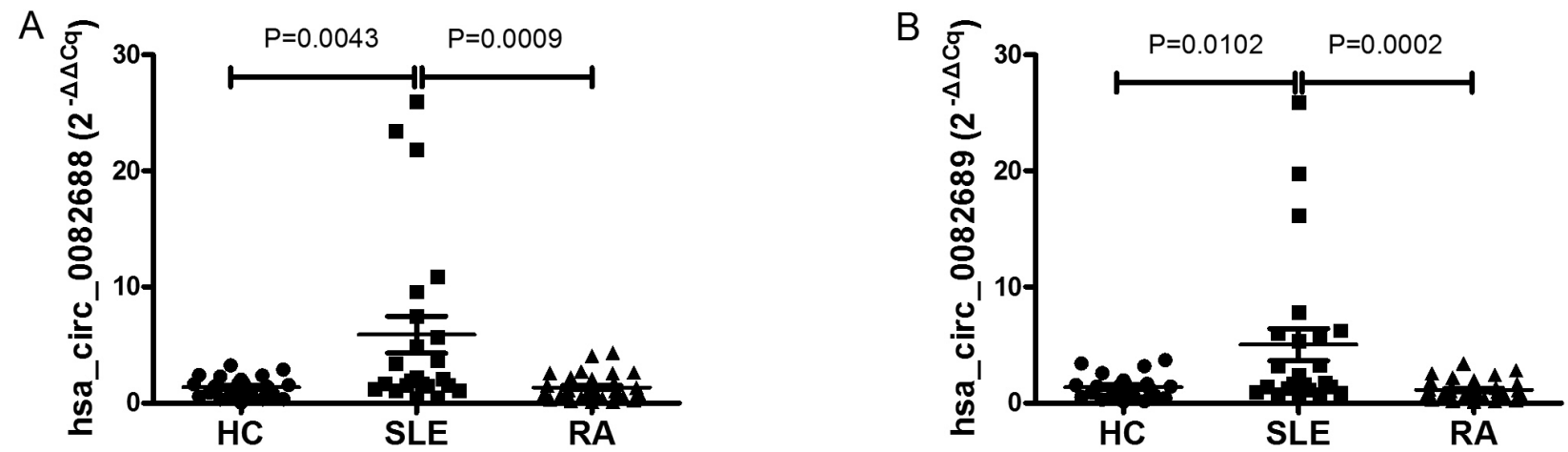

Figure 4. Validation the of hsa_circ_0082688 and hsa_circ_0082689 in the blind testing set in peripheral blood from the SLE, RA and HC groups. (A) Average expression of hsa_circ_0082688 in patients with SLE was significantly increased compared with the HC and RA groups. (B) Average expression of hsa circ_0082689 in patients with SLE was significantly increased compared with the HC and RA groups. HC, healthy controls; RA, rheumatoid arthritis; SLE, systemic lupus erythematosus.
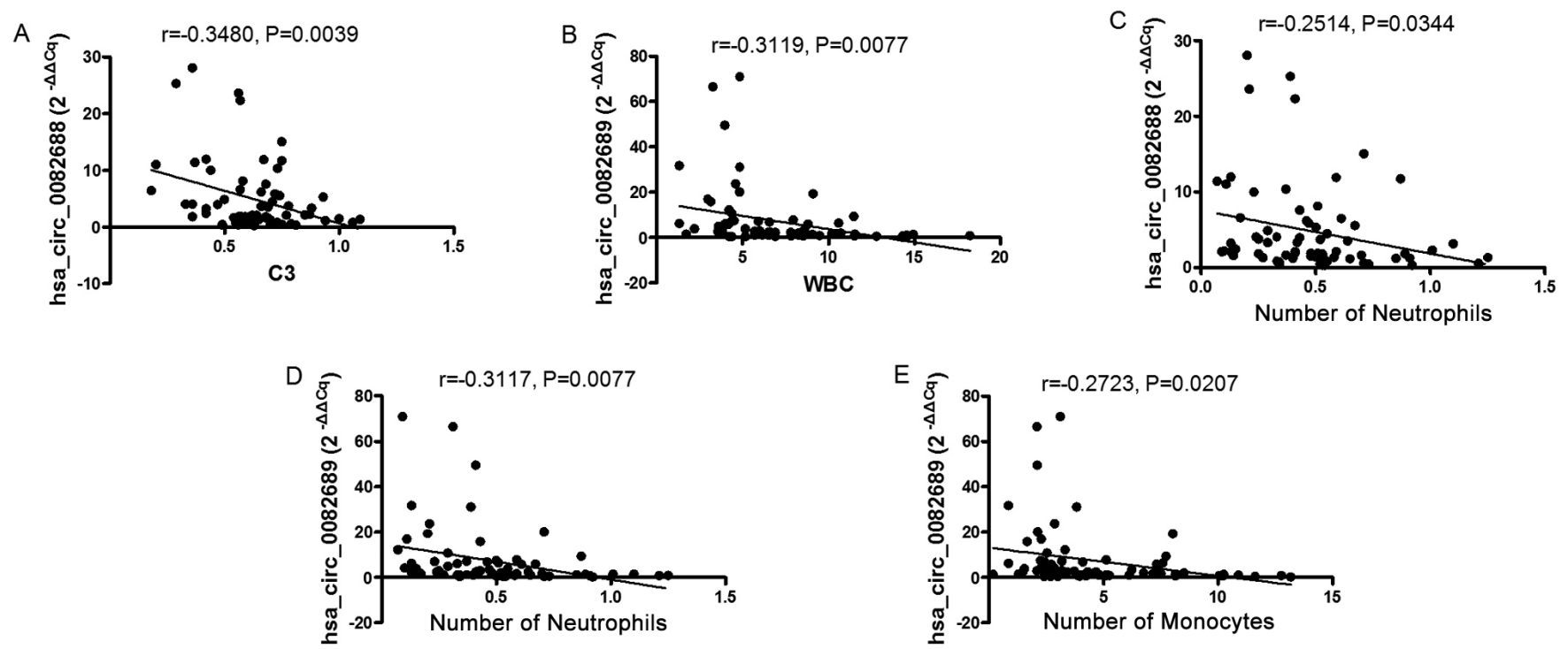

Figure 5. Correlation of the expression of identified circRNAs with SLE clinical characteristics. (A) Expression of hsa_circ_0082688 was negatively associated with C3 in patients with SLE. (B) Expression of hsa_circ_0082689 was negatively associated with WBC in patients with SLE. (C) Expression of hsa_circ_0082688 was negatively associated with the number of monocytes in patients with SLE. (D) Expression of hsa_circ_0082689 was negatively associated with the number of monocytes in patients with SLE. (E) Expression of hsa_circ_0082689 was negatively associated with number of neutrophils in patients with SLE. circRNAs, circular RNAs; SLE, systemic lupus erythematosus; WBC, white blood cell.

were calculated (Table III), and the correlations between this dataset and the expression levels of the specific differentially expressed circRNAs were analyzed. The results indicated that, while hsa_circ_0082688 expression was negatively associated with C3 in patients with SLE (r=-0.3480, P=0.0039; Fig. 5A), there was no correlation between the expression levels of the other confirmed circRNAs and C3 (data not shown). Furthermore, the expression levels of all identified circRNAs in the peripheral blood from patients with SLE did not correlate with SLEDAI, CRP, ESR or C4, which also reflect the severity of the disease (data not shown).

SLE is a complex autoimmune disease characterized by multiple organ system damages, such as the hematological system and skin. In the present study, the correlations between SLE-related clinical features and the expression levels of circRNAs were analyzed. It was demonstrated that the expression of hsa_circ_0082689 was negatively associated with white blood cell (WBC) number ( $\mathrm{r}=-0.3119, \mathrm{P}=0.0077$; Fig. 5B), monocyte number ( $\mathrm{r}=-0.3117, \mathrm{P}=0.0077$; Fig. 5D) and neutrophil number in patients with SLE ( $r=-0.2723, \mathrm{P}=0.0207$; Fig. 5E). In addition, the expression of hsa_circ_0082688 was negatively associated with monocytes number in patients with SLE ( $r=-0.2514$, P=0.0344; Fig. 5C). However, no significant difference was identified between the expression levels of circRNAs and other clinical features.

Production of multiple auto-antibodies, such as anti-dsDNA and anti-ENAs, is an important characteristic of SLE. Thus, the present study investigated the correlation between the expression levels of circRNAs and auto-antibodies in patients with SLE, but no significant difference was found (data not shown). However, all identified circRNAs did correlate with each other. For example, the expression of hsa_circ_0082688 correlated with the expression of hsa_circ_0082689 (r=0.5967; $\mathrm{P}<0.0001$; data not shown). 


\section{Discussion}

Previous studies have reported the feasibility of using circulating miRNAs and long non-coding (lnc) RNA as potential biomarkers of SLE $(9,34,35)$. Similar to miRNA and lncRNA, the potential of circulating circRNAs as powerful and non-invasive biomarkers in a number of diseases, including cancer (36), RA (19) and cardiovascular diseases (25), has been revealed. Compared with miRNAs and lncRNAs, circRNAs are more stable in mammalian cells (37) and their expression levels can be $\geq 10$-fold compared with those of their linear isomers (12). These properties indicate that the potential of circRNAs to be ideal biomarkers for human diseases (21). Moreover, the diagnostic performance of plasma or PBMC circRNA has been examined in SLE $(38,39)$. However, to the best of our knowledge, the expression profiles and diagnostic performance of circulating circRNAs in peripheral blood from patients with SLE have been rarely reported, and there was previously only one paper investigating circRNAs expression in peripheral blood from patients with SLE (40). In this study, Li et al (40) assessed the microarray profile of circRNAs in peripheral blood to identify the changes in the expression of circRNAs between pediatric patients with SLE and healthy children, and revealed that the expression levels of hsa_circ_0057762 and hsa_circ_0003090 could differentiate the pediatric patients with SLE from the healthy children. To the best of our knowledge, the present study was the first to performed a microarray analysis to investigate the changes in expression of circRNAs in peripheral blood from adult patients with SLE, by comparing with those in adult HCs. Microarray data identified a total of 1,566 circRNAs (753 were upregulated) that were significantly dysregulated in patients with SLE compared with HCs. Thus, it was speculated that circRNA may be a novel biomarkers for SLE diagnosis or disease process monitoring in adult patients. However, possibly due to age differences, the expression levels of hsa_circ_0057762 and hsa_circ_0003090 in peripheral blood were not significantly different between adult patients with SLE and HCs.

To determine whether differentially expressed circRNAs can be diagnostic biomarkers for SLE, three circRNAs hsa_circ_0082688, hsa_circ_0082689 and hsa_circ_0008675, which were listed in the top 50 most significant upregulated circRNAs in peripheral blood of patients with SLE in this circRNA microarray, and also identified to be upregulated in PBMCs from patients with SLE in our previous study (26), were chosen for validation by RT-qPCR in a training set. The results suggested that the expression levels of all three circRNAs increased significantly in patients with SLE. Furthermore, ROC curve analysis demonstrated that these circRNAs had the potential to distinguish between SLE and HCs. ROC curve analysis also indicated that the combination of hsa_circ_0082688 and hsa_circ_0082689 could provide the best diagnostic accuracy, with an AUC of 0.740. Moreover, the results from the further blind testing set suggested its good performance, not only in distinguishing between SLE and HCs groups, but also in distinguishing between patients with SLE and those with RA. Therefore, the present results indicated that this diagnostic model may be promising for SLE diagnosis.
In addition, the present results demonstrated that the combination model of hsa_circ_0082688-hsa_circ_0082689 + anti-dsDNA could more effectively discriminated the SLE group from the control groups ( $\mathrm{RA}+\mathrm{HC})$, with a sensitivity of $95.65 \%$ (22/23), a specificity of $100.00 \%(56 / 56)$ and an accuracy of $98.73 \%$ (78/79), which were superior to hsa_circ_0082688-hsa_circ_0082689 and anti-dsDNA. Collectively, it was speculated that the combination of circRNAs and traditional biomarkers could further improve the diagnostic value.

The field of circRNAs is recently discovered, and to the best of our knowledge, no previous study has definitively demonstrated the function of hsa_circ_0082688, hsa_circ_0082689 and hsa_circ_0008675. It has been reported that circRNAs can function as miRNA 'sponges' to sequester and competitively suppress miRNA activity. Moreover, their interaction with disease-associated miRNAs suggests that circRNAs are important for regulating diseases. Therefore, to investigate the possible function of these candidate circRNAs, the present study searched for potential miRNA targets of these circRNAs using Arraystar miRNA target prediction software, and numerous target miRNAs were identified. Among these target miRNAs, hsa-miR-506-3p, hsa-miR-127-5p and hsa-miR-153-3p were previously reported to be involved in the pathogenesis of SLE. For example, hsa-miR-506-3p has the potential to regulate the expression of Beclin1 (41), a protein that had been shown to regulate autophagy in SLE (42). In addition, hsa-miR-127-5p is involved in cell proliferation via the PI3K/Akt pathway (43), and thus may play a role in the senescence of mesenchymal stem cells and the development of SLE (44). hsa-miR-153-3p has also been reported to be involved in the development of lupus nephritis (45). Therefore, it was hypothesized that these candidate circRNAs, including hsa_circ_0082688, hsa_circ_0082689 and hsa_circ_0008675, may function in SLE pathogenesis.

The present study also investigated the correlations between the expression levels of these candidate circRNAs and the severity of SLE. The results demonstrated that, while the expression of hsa_circ_0082688 was negatively associated with C3 in patients with SLE, no other circRNAs expression levels correlated with C3. Moreover, the expression levels of all identified differentially expressed circRNAs in the peripheral blood from patients with SLE did not correlate with SLEDAI, CRP, ESR or C4. Thus, the results demonstrated the expression levels of hsa_circ_0082688, hsa_circ_0082689 and hsa_circ_0008675 were not potential biomarkers for the severity of SLE. Furthermore, the expression levels of these circRNAs between patients with newly diagnosed and recurrent SLE were not significantly different, and this also corroborates the aforementioned conclusion. However, it was found that the expression levels of hsa_circ_0082688,hsa_circ_0082689 and hsa_circ_0008675 were negatively associated with the total WBC number or the number of certain subclasses of WBC in patients with SLE, which indicated that the expression levels of hsa_circ_0082688, hsa_circ_0082689 and hsa_circ_0008675 in peripheral blood were associated with hematological system damage of SLE to some extent.

In addition, the present results suggested that the expressions levels of hsa_circ_0082688, hsa_circ_0082689 and hsa_circ_0008675 were correlated with each other, and similar 
findings have been reported in a previous study (23). Thus, these differentially expressed circRNAs may interact with each other directly or indirectly, although further experiments are required to test this hypothesis.

However, there are several limitations in this study that should be acknowledged. First is the relatively small sample size, especially the sample size of patients with newly diagnosed SLE, which may restrict the generalizability of the present results. Second, the exact role of these candidate circRNAs in SLE pathogenesis was not investigated in this study. Therefore, these are focuses of future studies.

In conclusion, to the best of our knowledge, the present study was the first to demonstrate the circRNA expression profiles in peripheral blood from adult patients with SLE, and identify that circRNAs may serve as novel biomarkers for SLE diagnosis. Furthermore, it was found that the combination of hsa_circ_0082688 and hsa_circ_0082689 had a relatively good capacity in discriminating the SLE groups from both HCs and RA groups. Therefore, the present results provide a novel potential diagnostic biomarker for SLE diagnosis, and may facilitate improved understanding of the hematological system damage of SLE.

\section{Acknowledgements}

The authors would like to acknowledge the help from Dr RuiWu from the Department of Rheumatology, The First Affiliated Hospital of Nanchang University (Nanchang, China).

\section{Funding}

This study was supported by the Science and Technology Plan Project of the Education Department of Jiangxi Province (grant no. GJJ170008), the National Natural Science Foundation of China (grant nos. 81360459, 81560344 and 81660277), the Jiangxi Provincial Natural Science Foundation of China (grant nos. 20171BAB205113 and 20171ACB20032), the Science and Technology Project of Health and Family Planning Commission of Jiangxi Province of China (grant no. 20165094) and the Foundation for Distinguished Young Scientists of Jiangxi Province of China (grant no. 20171BCB23087).

\section{Availability of data and materials}

The datasets used and/or analyzed during the current study are available from the corresponding author on reasonable request.

\section{Authors' contributions}

JL and QL conceived the project and wrote the study. JL, QL, $\mathrm{XL}$ and $\mathrm{ZH}$ designed the experiments. JL, QL, XL, LZ and BF collected the study subjects, performed the experiments and analyzed the data. QL, XL, LZ, CQ, LF, YG and ZH performed the experiments. All authors read and approved the final manuscript.

\section{Ethics approval and consent to participate}

The present study was authorized by the Ethics Committee of The First Affiliated Hospital of Nanchang University. All participants provided written informed consent prior to the initiation of the study.

\section{Patient consent for publication}

Not applicable.

\section{Competing interests}

The authors declare that they have no competing interests.

\section{References}

1. Tsokos GC: Systemic lupus erythematosus. N Engl J Med 365: 2110-2121, 2011.

2. Ruiz-Irastorza G, Ramos-Casals M, Brito-Zeron P and Khamashta MA: Clinical efficacy and side effects of antimalarials in systemic lupus erythematosus: A systematic review. Ann Rheum Dis 69: 20-28, 2010.

3. Chang NH, Li TT, Kim JJ, Landolt-Marticorena C, Fortin PR, Gladman DD, Urowitz MB and Wither JE: Interferon alpha induces altered transitional B cell signaling and function in systemic lupus erythematosus. J Autoimmun 58: 100-110, 2015.

4. Zhao M, Liu S, Luo S, Wu H, Tang M, Cheng W, Zhang Q, Zhang P, Yu X, Xia Y, et al: DNA methylation and mRNA and microRNA expression of SLE CD4+ T cells correlate with disease phenotype. J Autoimmun 54: 127-136, 2014.

5. O'Gorman WE, Hsieh EW, Savig ES, Gherardini PF, Hernandez JD, Hansmann L, Balboni IM, Utz PJ, Bendall SC, Fantl WJ, et al: Single-cell systems-level analysis of human Toll-like receptor activation defines a chemokine signature in patients with systemic lupus erythematosus. J Allergy Clin Immunol 136: 1326-1336, 2015.

6. Lech M, Kantner C, Kulkarni OP, Ryu M, Vlasova E, Heesemann J, Anz D, Endres S, Kobayashi KS, Flavell RA, et al: Interleukin-1 receptor-associated kinase-M suppresses systemic lupus erythematosus. Ann Rheum Dis 70: 2207-2217, 2011.

7. Huang Z, Shi Y, Cai B, Wang L, Wu Y, Ying B, Qin L, Hu C and $\mathrm{Li}$ Y: MALDI-TOF MS combined with magnetic beads for detecting serum protein biomarkers and establishment of boosting decision tree model for diagnosis of systemic lupus erythematosus. Rheumatology (Oxford) 48: 626-631, 2009.

8. Ferreira TAR, de Andrade HM, de Pádua PM, Carvalho MDG, Pires SDF, Oliveira IHR, Lima BSS, Fialho Júnior LC, Cicarini WB, Chapeourouge DA, et al: Identification of potential biomarkers for systemic lupus erythematosus diagnosis using two-dimensional differential gel electrophoresis (2D-DIGE) and mass spectrometry. Autoimmunity 50: 247-256, 2017.

9. Wu GC, Li J, Leng RX, Li XP, Li XM, Wang DG, Pan HF and Ye DQ: Identification of long non-coding RNAs GAS5, linc0597 and lnc-DC in plasma as novel biomarkers for systemic lupus erythematosus. Oncotarget 8: 23650-23663, 2017.

10. Barrett SP, Wang PL and Salzman J: Circular RNA biogenesis can proceed through an exon-containing lariat precursor. Elife 4: e07540, 2015.

11. Li Z, Huang C, Bao C, Chen L, Lin M, Wang X, Zhong G, Yu B, $\mathrm{Hu}$ W, Dai L, et al: Exon-intron circular RNAs regulate transcription in the nucleus. Nat Struct Mol Biol 22: 256-264, 2015.

12. Jeck WR, Sorrentino JA, Wang K, Slevin MK, Burd CE, Liu J, Marzluff WF and Sharpless NE: Circular RNAs are abundant, conserved, and associated with ALU repeats. RNA 19: 141-157, 2013.

13. Memczak S, Jens M, Elefsinioti A, Torti F, Krueger J, Rybak A, Maier L, Mackowiak SD, Gregersen LH, Munschauer M, et al: Circular RNAs are a large class of animal RNAs with regulatory potency. Nature 495: 333-338, 2013.

14. Hansen TB, Jensen TI, Clausen BH, Bramsen JB, Finsen B, Damgaard CK and Kjems J: Natural RNA circles function as efficient microRNA sponges. Nature 495: 384-388, 2013.

15. Jeck WR and Sharpless NE: Detecting and characterizing circular RNAs. Nat Biotechnol 32: 453-461, 2014.

16. Burd CE, Jeck WR, Liu Y, Sanoff HK, Wang Z and Sharpless NE: Expression of linear and novel circular forms of an INK4/ARF-associated non-coding RNA correlates with atherosclerosis risk. PLoS Genet 6: e1001233, 2010.

17. Hansen TB, Kjems J and Damgaard CK: Circular RNA and miR-7 in cancer. Cancer Res 73: 5609-5612, 2013. 
18. Li F, Zhang L, Li W, Deng J, Zheng J, An M, Lu J and Zhou Y: Circular RNA ITCH has inhibitory effect on ESCC by suppressing the Wnt/ $\beta$-catenin pathway. Oncotarget 6: 6001-6013, 2015.

19. Ouyang Q, Wu J, Jiang Z, Zhao J, Wang R, Lou A, Zhu D, Shi GP and Yang M: Microarray expression profile of circular RNAs in peripheral blood mononuclear cells from rheumatoid arthritis patients. Cell Physiol Biochem 42: 651-659, 2017.

20. Lin X, Lo HC, Wong DT and Xiao X: Noncoding RNAs in human saliva as potential disease biomarkers. Front Genet 6: 175, 2015.

21. Memczak S, Papavasileiou P, Peters O and Rajewsky N: Identification and characterization of circular RNAs As a new class of putative biomarkers in human blood. PLoS One 10 e0141214, 2015.

22. You X, Vlatkovic I, Babic A, Will T, Epstein I, Tushev G, Akbalik G, Wang M, Glock C, Quedenau C, et al: Neural circular RNAs are derived from synaptic genes and regulated by development and plasticity. Nat Neurosci 18: 603-610, 2015.

23. Salzman J, Chen RE, Olsen MN, Wang PL and Brown PO: Cell-type specific features of circular RNA expression. PLoS Genet 9: e1003777, 2013

24. Zhao Z, Li X, Jian D, Hao P, Rao L and Li M: Hsa_circ_0054633 in peripheral blood can be used as a diagnostic biomarker of pre-diabetes and type 2 diabetes mellitus. Acta Diabetol 54: 237-245, 2017

25. Zhao Z, Li X, Gao C, Jian D, Hao P, Rao L and Li M: Peripheral blood circular RNA hsa_circ_0124644 can be used as a diagnostic biomarker of coronary artery disease. Sci Rep 7: 39918, 2017.

26. Luo Q,Zhang L, LiX, Fu B, Guo Y,Huang Zand Li J: Identification of circular RNAs hsa_circ_0044235 and hsa_circ_0068367 as novel biomarkers for systemic lupus erythematosus. Int J Mol Med 44: 1462-1472, 2019.

27. Hochberg MC: Updating the American college of rheumatology revised criteria for the classification of systemic lupus erythematosus. Arthritis Rheum 40: 1725, 1997.

28. Bombardier C, Gladman DD, Urowitz MB, Caron D and Chang CH: Derivation of the SLEDAI. A disease activity index for lupus patients. The committee on prognosis studies in SLE. Arthritis Rheum 35: 630-640, 1992.

29. Arnett FC, Edworthy SM, Bloch DA, McShane DJ, Fries JF, Cooper NS, Healey LA, Kaplan SR, Liang MH, Luthra HS, et al: The American rheumatism association 1987 revised criteria for the classification of rheumatoid arthritis. Arthr Rhuem 31 315-324, 1988

30. Luo Q, Li X, Xu C, Zeng L, Ye J, Guo Y, Huang Z and Li J: Integrative analysis of long non-coding RNAs and messenger RNA expression profiles in systemic lupus erythematosus. Mol Med Rep 17: 3489-3496, 2018.

31. Livak KJ and Schmittgen TD: Analysis of relative gene expression data using real-time quantitative PCR and the 2(-Delta Delta C(T)) method. Methods 25: 402-408, 2001.

32. Luo Q, Zhang L, Fang L, Fu B, Guo Y, Huang Z and Li J: Circular RNAs hsa_circ_0000479 in peripheral blood mononuclear cells as novel biomarkers for systemic lupus erythematosus. Autoimmunity 53: 167-176, 2020.

33. Soliman S and Mohan C: Lupus nephritis biomarkers. Clin Immunol 185: 10-20, 2017.
34. Stypinska B and Paradowska-Gorycka A: Cytokines and MicroRNAs as candidate biomarkers for systemic lupus erythematosus. Int J Mol Sci 16: 24194-24218, 2015.

35. Wu Y, Zhang F, Ma J, Zhang X, Wu L, Qu B, Xia S, Chen S, Tang Y and Shen N: Association of large intergenic noncoding RNA expression with disease activity and organ damage in systemic lupus erythematosus. Arthritis Res Ther 17: 131, 2015.

36. Guo JN, Li J, Zhu CL, Feng WT, Shao JX, Wan L, Huang MD and He JD: Comprehensive profile of differentially expressed circular RNAs reveals that hsa_circ_0000069 is upregulated and promotes cell proliferation, migration, and invasion in colorectal cancer. Onco Targets Ther 9: 7451-7458, 2016.

37. Liang D and Wilusz JE: Short intronic repeat sequences facilitate circular RNA production. Genes Dev 28: 2233-2247, 2014.

38. Ouyang Q, Huang Q, Jiang Z, Zhao J, Shi GP and Yang M: Using plasma circRNA_002453 as a novel biomarker in the diagnosis of lupus nephritis. Mol Immunol 101: 531-538, 2018.

39. Zhang MY, Wang JB, Zhu ZW, Li LJ, Liu RS, Yang XK, Leng RX, Li XM, Pan HF and Ye DQ: Differentially expressed circular RNAs in systemic lupus erythematosus and their clinical significance. Biomed Pharmacother 107: 1720-1727, 2018.

40. Li S, Zhang J, Tan X, Deng J, Li Y, Piao Y, Li C, Yang W, Mo W, Sun J, et al: Microarray expression profile of circular RNAs and mRNAs in children with systemic lupus erythematosus. Clin Rheumatol 38: 1339-1350, 2019.

41. Yi F, Hao Y, Chong X and Zhong W: Overexpression of microRNA-506-3p aggravates the injury of vascular endothelial cells in patients with hypertension by downregulating Beclin1 expression. Exp Ther Med 15: 2844-2850, 2018.

42. Pan Q, Gao C, Chen Y, Feng Y, Liu WJ and Liu HF: Update on the role of autophagy in systemic lupus erythematosus: A novel therapeutic target. Biomed Pharmacother 71: 190-193, 2015.

43. Liang J, Xu L, Zhou F, Liu AM, Ge HX, Chen YY and Tu M: MALAT1/miR-127-5p regulates osteopontin (OPN)-mediated proliferation of human chondrocytes through $\mathrm{PI} 3 \mathrm{~K} / \mathrm{Akt}$ pathway. J Cell Biochem 119: 431-439, 2018.

44. Chen H, Shi B, Feng X, Kong W, Chen W, Geng L, Chen J, Liu R, Li X, Chen W, et al: Leptin and neutrophil-activating peptide 2 promote mesenchymal stem cell senescence through activation of the phosphatidylinositol 3-kinase/Akt pathway in patients with systemic lupus erythematosus. Arthritis Rheumatol 67: 2383-2393, 2015

45. Navarro-QuirozE,Pacheco-LugoL,Navarro-QuirozR,LorenziH, España-Puccini P, Díaz-Olmos Y, Almendrales L, Olave V, Gonzalez-Torres H, Diaz-Perez A, et al: Profiling analysis of circulating microRNA in peripheral blood of patients with class IV lupus nephritis. PLoS One 12: e0187973, 2017.

This work is licensed under a Creative Commons Attribution-NonCommercial-NoDerivatives 4.0 International (CC BY-NC-ND 4.0) License. 